\title{
Inter-comparison of Hauser-Feshbach model codes toward better actinide evaluations
}

\author{
Roberto Capote ${ }^{1}$, Stéphane Hilaire ${ }^{2}$, Osamu Iwamoto ${ }^{3}$, Toshihiko Kawano ${ }^{4, a}$, and Mihaela Sin $^{5}$ \\ 1 NAPC-Nuclear Data Section, International Atomic Energy Agency, Vienna 1400, Austria \\ 2 CEA, DAM, DIF, 91297 Arpajon, France \\ 3 Nuclear Data Center, Japan Atomic Energy Agency, Tokai-mura, Ibaraki 319-1195, Japan \\ 4 Theoretical Division, Los Alamos National Laboratory, Los Alamos, New Mexico 87545, USA \\ 5 Faculty of Physics, University of Bucharest, Bucharest-Magurele, Romania
}

\begin{abstract}
The Hauser-Feshbach codes, EMPIRE, TALYS, CCONE, and $\mathrm{CoH}_{3}$, which are widely utilized in nuclear data evaluations, are compared, with a particular focus on neutron-induced reactions on major actinides. We report the results of Hauser-Feshbach calculations using well-defined input parameters, and discuss the differences among these codes.
\end{abstract}

\section{Introduction}

The Hauser-Feshbach (HF) codes that include a preequilibrium model have been playing for years a central role in producing evaluated nuclear reaction data files. The codes, such as EMPIRE [1], TALYS [2], CCONE [3], and $\mathrm{CoH}_{3}$ [4], have been and are still actively upgraded in order to better understand nuclear reaction mechanisms by sharing and exchanging theoretical knowledge as well as computational techniques among actors involved in their development. Albeit the framework of the model codes aforementioned is quite similar, implementation of the reaction models as well as computational techniques adopted may produce some differences in the calculated results. The International Atomic Energy Agency (IAEA) Nuclear Data Section conducts one of such interorganizational collaborations, and defined some exercises to compare the HF codes developed for nuclear data evaluations, with a particular focus on the actinide evaluations. Watanabe et al. [5] performed a similar comparison, including EMPIRE, TALYS, and $\mathrm{CoH}$ (ver. $2)$. In this paper we report the results of HF calculations for neutron induced reactions on ${ }^{238} \mathrm{U}$ and ${ }^{239} \mathrm{Pu}$ employing the input parameters defined in the IAEA report [6].

For performing cross section calculations, reaction mechanisms other than the compound nuclear reaction are often involved, such as the direct reactions, the direct/semidirect capture process, the pre-equilibrium model, and so on. In order to minimize the influence coming from implementation of these peripheral models, we conduct our code comparison in the fast energy range, where the compound nuclear reactions with the coupledchannels (CC) optical model play the major role. In case the HF codes provide several options that control the calculation, we choose the most commonly used one. In other words, we perform a default calculation, except parameters specifically given in the IAEA report [6]. It

a e-mail: kawano@lanl.gov is shown [7] that inclusion of direct reaction channels changes the compound elastic and inelastic scattering cross sections. However, we do not include the so-called Engelbrecht-Weidenmüller transformation [8], since this option in many of the HF codes is not yet operational.

\section{Code comparisons}

\subsection{Overview}

A brief summary of the HF codes in this study is given in Table 1. Although there are more HF codes available, in particular, commonly used in the nuclear astrophysics community, or other pioneering codes for nuclear data evaluations such as GNASH [9], we limit ourselves to the 4 codes.

- The first version of the EMPIRE code was developed by M. Herman around 1980 at Warsaw (Poland), and was intended for interpretation of activation measurements. In 1990 the code has been entirely rewritten to modernize its structure. The EMPIRE code is actively updated by its developing team (M. Herman, R. Capote, A. Trkov, M. Sin, and B.V. Carlson) and by other contributors through the GForge collaboration server at BNL.

- The TALYS code development started in 1997 within a collaboration between NRG Petten and CEA. The current core development team consists of A. Koning, S. Hilaire, and S. Goriely. All the nuclear reactions models required to describe light particle induced nuclear reaction from $1 \mathrm{keV}$ up to $200 \mathrm{MeV}$ are implemented. A large nuclear structure database, as well as the use of systematics otherwise, enables a minimal input file to be used to provide a default output.

- The CCONE code was developed by O. Iwamoto of JAEA for nuclear data evaluation of JENDL Actinide File 2008 and JENDL-4.0. It consists of

(C) The Authors, published by EDP Sciences. This is an Open Access article distributed under the terms of the Creative Commons Attribution License 4.0 (http://creativecommons.org/licenses/by/4.0/). 
Table 1. The Hauser-Feshbach Codes for nuclear data evaluation.

\begin{tabular}{lcrl}
\hline & lang. & lines & maintained by \\
\hline EMPIRE & Fortran77/90 & $113 \mathrm{~K}$ & BNL/IAEA \\
TALYS & Fortran77/90 & $118 \mathrm{~K}$ & IAEA/CEA/ULB \\
CCONE & $\mathrm{C}++$ & $35 \mathrm{~K}$ & JAEA \\
$\mathrm{CoH}_{3}$ & $\mathrm{C}++$ & $46 \mathrm{~K}$ & LANL \\
\hline
\end{tabular}

CC optical model, pre-equilibrium exciton model and HF statistical models. The applicable energy range was extended later for the JENDL-4.0/HE development.

- The CC and HF code, $\mathrm{CoH}$, was first released in 1992, developed by T. Kawano in Kyushu Univ. The original code was limited to binary reactions only, and it was extended to calculate general nuclear reaction cross sections in 2009.

All these codes are capable of performing reaction cross section calculations for deformed systems by applying the $\mathrm{CC}$ model. The transmission coefficients, with a correction of the direct reaction channel [8], are fed to the HF main part, and the width fluctuation correction (WFC) is performed. Each code adopts different WFC, namely TALYS and CCONE employ the WFC model by Moldauer [10], EMPIRE has the model of Hofmann et al. [11,12]. The WFC in $\mathrm{CoH}_{3}$ is based on Moldauer's model with a new parameterization based on the Gaussian Orthogonal Ensemble (GOE) [7, 13]. Hilaire et al. demonstrated the difference in the WFC models adopted [14].

For performing the code comparison, we adopt model input parameters as consistent as possible, and sometimes this requires ad-hoc modifications to the source codes. We employ a relatively simple parameterization to minimize such code modifications and to facilitate the comparison. Therefore, the calculated results are not necessarily reproducing the available experimental or evaluated data. The focus is to find differences among the codes due to different coding and implementation by using exactly the same controlled set of input parameters.

\subsection{Coupled-channels optical model}

EMPIRE and TALYS invoke internally the CC code ECIS [15] to perform the optical model calculations, while CCONE and $\mathrm{CoH}_{3}$ have their own CC equation solver. We expect the code difference owing to the $\mathrm{CC}$ calculation to be negligible. Table 2 shows calculated total $\left(\sigma_{\mathrm{T}}\right)$, the shape elastic $\left(\sigma_{\mathrm{SE}}\right)$, and the compound formation $\left(\sigma_{\mathrm{CN}}\right)$ cross sections for the neutron induced reactions on ${ }^{238} \mathrm{U}$ at $100 \mathrm{keV}$. The optical potential parameters of Soukhovitskii et al. [16] are used, and five levels $\left(0^{+}, 2^{+}, 4^{+}, 6^{+}\right.$, and $8^{+}$) of the ground state rotational band are coupled. The largest difference is at most $0.7 \%$ seen in $\sigma_{\mathrm{CN}}$. Such a good agreement amongst different $\mathrm{CC}$ codes is also reported elsewhere [17]. Although we show the comparison at $100 \mathrm{keV}$ only, in general the agreement is improved as the incident energy increases.

One of the reasons of the modest differences in the optical model calculation comes from an energy dependence of the potential parameters. For example, there is an ambiguity in calculating the coupling potential between different energy states when the optical potential is energy-dependent. Although this is not well-defined in
Table 2. Calculated optical model cross sections in barns, $\sigma_{\mathrm{T}}$ total, $\sigma_{\mathrm{SE}}$ shape elastic, and $\sigma_{\mathrm{CN}}$ compound formation cross sections for the $100-\mathrm{keV}$ neutron induced reaction on ${ }^{238} \mathrm{U}$ with the coupled-channels model codes, ECIS, CCONE, and $\mathrm{CoH}_{3}$.

\begin{tabular}{lllll}
\hline & $\sigma_{\mathrm{T}}$ & $\sigma_{\mathrm{SE}}$ & $\sigma_{\mathrm{CN}}$ & \\
\hline ECIS & 12.35 & 9.236 & 3.070 & \\
CCONE & 12.37 & 9.242 & 3.091 & \\
$\mathrm{CoH}_{3}$ & 12.33 & 9.213 & 3.080 & {$[\mathrm{~b}]$} \\
\hline
\end{tabular}
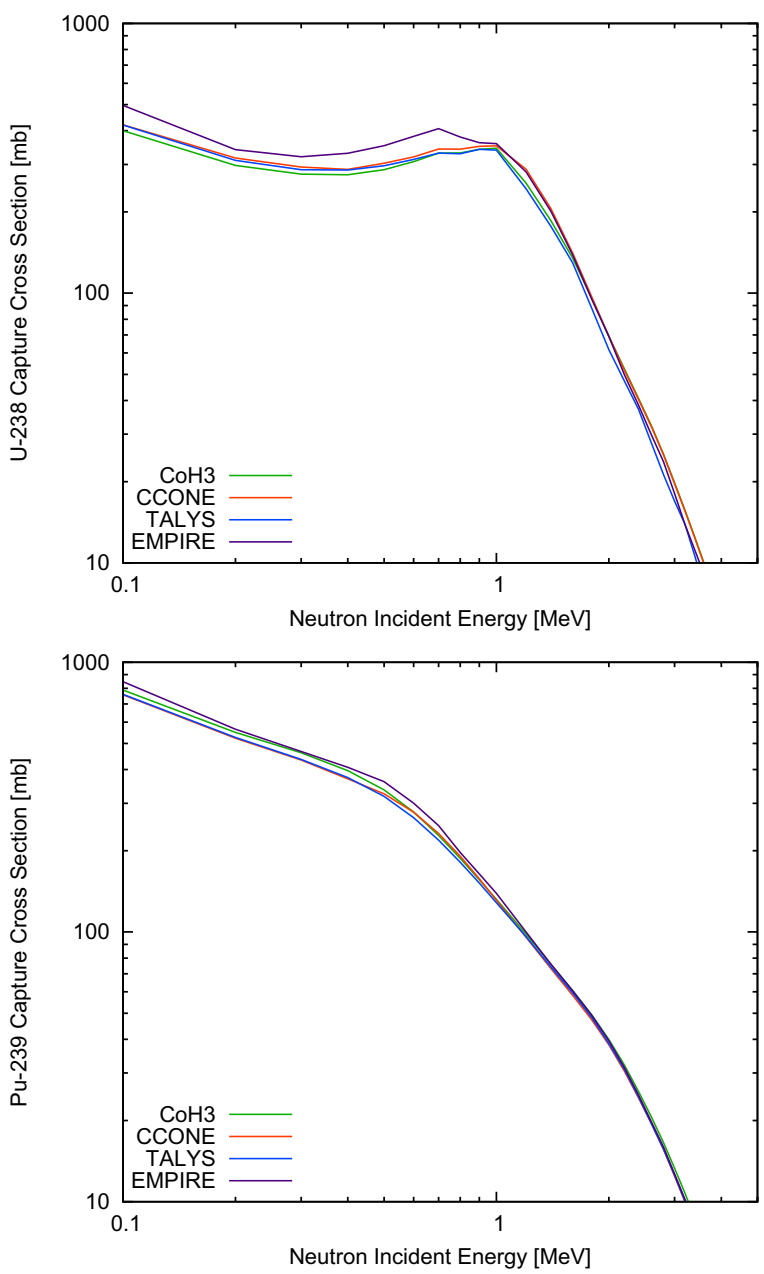

Figure 1. Comparison of calculated neutron radiative capture cross sections for ${ }^{238} \mathrm{U}$ (top panel) and ${ }^{239} \mathrm{Pu}$ (bottom panel).

our case, the impact will be totally negligible when the particle incident energy is much larger than the inelastic channel threshold energies.

\subsection{Statistical Hauser-Feshbach model with width fluctuation correction}

The photon strength functions for E1 giant dipole resonance are calculated using a standard Lorentzian form, with the parameters given in Ref. [6]. The discrete level schemes are taken from RIPL-3 up to the same cut-off energies. For the level densities for ${ }^{238,239} \mathrm{U}$ and ${ }^{239,240} \mathrm{Pu}$ we adopt the microscopic calculations based on the Hartree-Fock-Bogoliubov (HFB) and combinatorial method [18], in the tabulated form, and interpolate if necessary. The level densities on top of the fission barriers are also taken from the HFB table. Since we need, for the inner barrier, an enhancement factor for the fission 

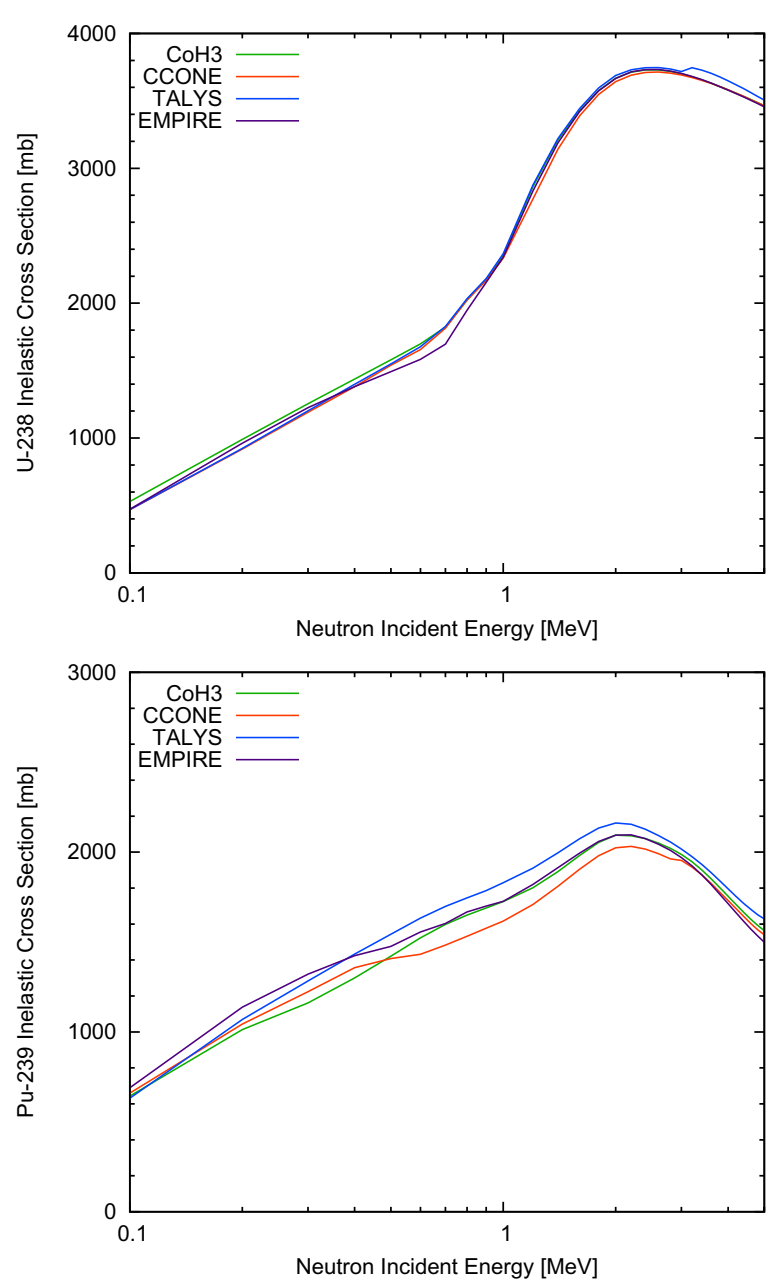

Figure 2. Comparison of calculated inelastic scattering cross sections for ${ }^{238} \mathrm{U}$ (top panel) and ${ }^{239} \mathrm{Pu}$ (bottom panel).

level density, and each code has a different prescription to calculate the enhancement, we employ a simple estimate for the latter assuming it to be constant to make sure that our fission level densities are the same. Assuming the deformation of $\beta_{2}=0.6$ and the temperature of $T=$ $0.3 \mathrm{MeV}$, the enhancement factor $K$ for the inner barrier reads

$$
K=\sqrt{\frac{\pi}{2} \sigma},
$$

where

$$
\sigma=0.01448 T A^{5 / 3}\left(1-\frac{2}{3} \beta_{2}\right),
$$

while $K=1$ for the outer barrier since the HFB table already account for the known broken left-right asymmetry on top of the outer barrier.

The calculated capture cross sections with the compared HF codes are shown in Fig 1. The maximum difference of $20 \%$ is seen at low energies. We assume there is less ambiguity in the adopted photon strength functions and the level density. The difference may occur due to the difference in the neutron transmission coefficients and the width fluctuation models. For example, albeit we employ the same optical potential parameters, EMPIRE, TALYS, and CCONE pre-generate the transmission coefficient array on a fixed energy grid and interpolate them to calculate the transmission to the excited states, while $\mathrm{CoH}_{3}$
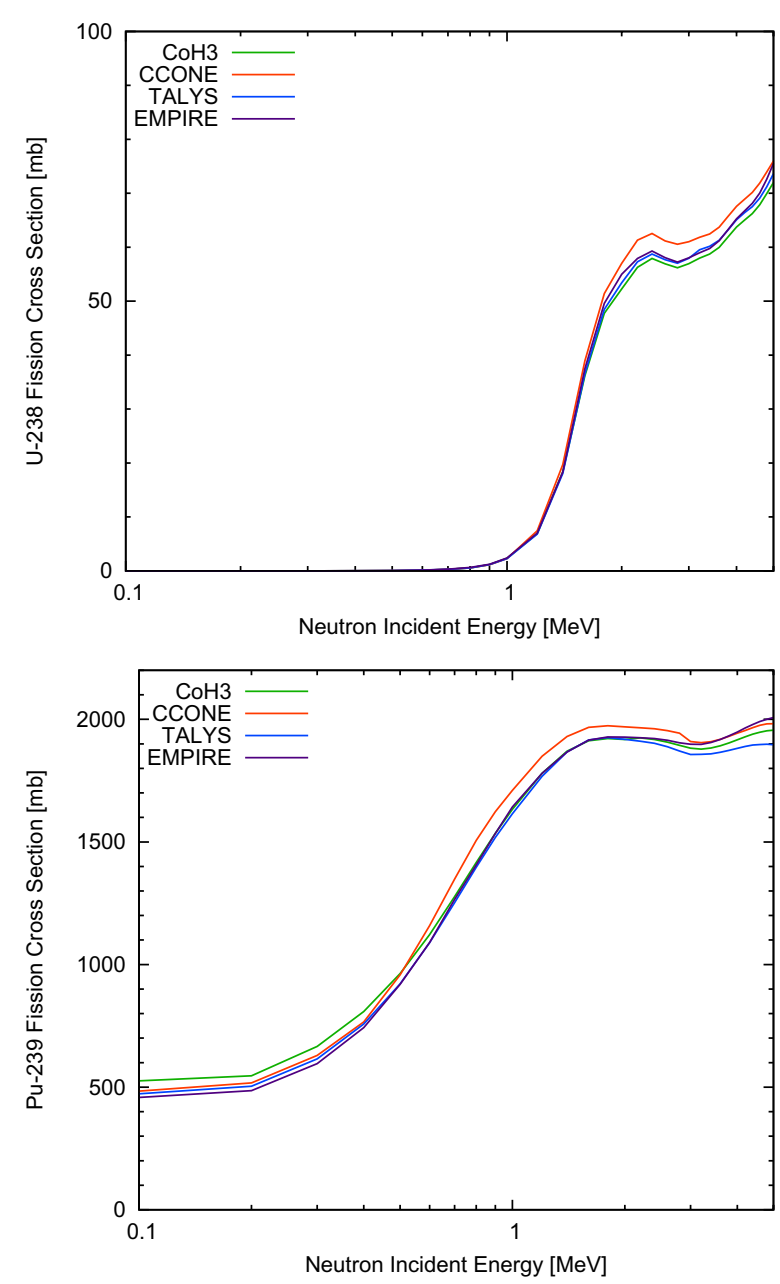

Figure 3. Comparison of calculated fission cross sections for ${ }^{238} \mathrm{U}$ (top panel) and ${ }^{239} \mathrm{Pu}$ (bottom panel).

generates them by the CC $S$-matrix [8]. This has some impact on the neutron channel at low energies, which is shown in Fig. 2, especially for the ${ }^{238} \mathrm{U}$ case.

Generally speaking, the agreement is better for the non-fissile case $\left({ }^{238} \mathrm{U}\right)$, which suggests that the difference in the fission channel modelling in each code produces differences in the calculated fission cross sections, and though competition, in other non-fission statistical channels. In this comparison we consistently employ the simplest Hill-Wheeler form [19] for calculating the fission transmission coefficients. The double-humped fission barrier is approximated by two inverted parabolas characterized by heights and curvatures $\left(V_{A}, \hbar \omega_{A}\right.$ for the inner barrier and $V_{B}, \hbar \omega_{B}$ for the outer barrier). The fission transmission coefficient for each barrier is given by

$$
T_{i}(E)=\frac{1}{1+\exp \left(2 \pi \frac{\left.V_{i}+E-E_{0}\right)}{\hbar \omega_{i}}\right)}, \quad i=A, B
$$

where $E_{0}$ is the excitation energy of the compound nucleus, $E$ is the nuclear excitation energy measured from the top of the barrier. The net fission transmission coefficient corresponds to the full damping approximation (independent barriers) [20] and is given by

$$
T_{f}=\frac{T_{A} T_{B}}{T_{A}+T_{B}}
$$


The HF codes in this comparison produce the fission cross sections shown in Fig. 3. The disagreement in the ${ }^{239} \mathrm{Pu}(\mathrm{n}, \mathrm{f})$ reaction is at most $15 \%$; $\mathrm{CoH}_{3}$ tends to be high at low energies, while CCONE gives the largest cross sections above $500 \mathrm{keV}$. It should be noted that the fission transmission coefficient varies rapidly when the energy difference $V_{i}+E-E_{0}$ in Eq. (3) approaches to zero. We expect some differences caused by different coding to be magnified by the high sensitivity in the fission transmission calculation. The coding differences include binning of the continuum, interpolation of the transmission coefficients or level densities, determination of the largest spin in the entrance channel, and so on. Advanced treatments of fission penetrability $[20,21]$ also modify the fission cross section calculations. However, we would say that the calculated cross sections with EMPIRE, TALYS, CCONE, and $\mathrm{CoH}_{3}$ agree within about $10 \%$ in most cases.

\section{Conclusion}

We calculated neutron-induced reaction cross sections on ${ }^{238} \mathrm{U}$ and ${ }^{239} \mathrm{Pu}$ in the fast energy range with the HauserFeshbach codes, EMPIRE, TALYS, CCONE, and $\mathrm{CoH}_{3}$, and compared the calculated cross sections. The coupledchannels calculation in each code produced almost identical cross sections. Meanwhile we demonstrated that the statistical model calculation is very sensitive to the fission channel, and that the calculated cross sections could be slightly different, particularly for the fissile case, despite the same input parameters are provided. However, the discrepancy is less than $10 \%$.

One of the authors (TK) carried out this work under the auspices of the National Nuclear Security Administration of the U.S. Department of Energy at Los Alamos National Laboratory under Contract No. DE-AC52-06NA25396.

\section{References}

[1] M. Herman, R. Capote, B. Carlson, P. Oblozinský, M. Sin, A. Trkov, H. Wienke, V. Zerkin, Nuclear Data Sheets 108, 2655 (2007)

[2] A.J. Koning, S. Hilaire, M.C. Duijvestijn, EPJ Web of Conferences pp. 211-214 (2008), proc. Int. Conf. on Nuclear Data for Science and Technology,
22 - 27 Apr., 2007, Nice, France, Ed. O. Bersillon, F. Gunsing, E. Bauge, R. Jacqmin, and S. Leray

[3] O. Iwamoto, Journal of Nuclear Science and Technology 44, 687 (2007)

[4] T. Kawano, P. Talou, M.B. Chadwick, T. Watanabe, Journal of Nuclear Science and Technology 47, 462 (2010)

[5] T. Watanabe, T. Kawano, M.B. Chadwick, R.O. Nelson, S. Hilaire, E. Bauge, P. Dossantos-Uzarralde, J. Nucl. Sci. Technol. 47, 470 (2010)

[6] R. Capote Noy, S. Goriely, S. Hilaire, O. Iwamoto, T. Kawano, A. Koning, Tech. Rep. INDC(NDS)0654, International Atomic Energy Agency (2014)

[7] T. Kawano, P. Talou, H.A. Weidenmüller, Phys. Rev. C 92, 044617 (2015)

[8] T. Kawano, R. Capote, S. Hilaire, P. Chau Huu-Tai, Phys. Rev. C 94, 014612 (2016)

[9] P.G. Young, E.D. Arthur, Tech. Rep. LA-6947, Los Alamos National Laboratory (1977)

[10] P.A. Moldauer, Nuclear Physics A 344, 185 (1980)

[11] H. Hofmann, J. Richert, J. Tepel, H. Weidenmüller, Annals of Physics 90, 403 (1975)

[12] H.M. Hofmann, T. Mertelmeier, M. Herman, J.W. Tepel, Zeitschrift für Physik A Atoms and Nuclei 297, 153 (1980)

[13] T. Kawano, P. Talou, Nuclear Data Sheets 118, 183 (2014)

[14] S. Hilaire, C. Lagrange, A.J. Koning, Annals of Physics 306, 209 (2003)

[15] J. Raynal, Tech. Rep. IAEA-SMR-9/8 (1972), iCTP International Seminar Course: Computing as a language of physics, Trieste, Italy, August 2-20 1971

[16] E.S. Soukhovitskii, S. Chiba, J.Y. Lee, O. Iwamoto, T. Fukahori, Journal of Physics G: Nuclear and Particle Physics 30, 905 (2004)

[17] F.S. Dietrich, I.J. Thompson, T. Kawano, Phys. Rev. C 85, 044611 (2012)

[18] S. Goriely, S. Hilaire, A.J. Koning, Phys. Rev. C 78, 064307 (2008)

[19] D.L. Hill, J.A. Wheeler, Phys. Rev. 89, 1102 (1953)

[20] M. Sin, R. Capote, M.W. Herman, A. Trkov, Phys. Rev. C 93, 034605 (2016)

[21] S. Goriely, S. Hilaire, A.J. Koning, M. Sin, R. Capote, Phys. Rev. C 79, 024612 (2009) 